$\begin{array}{cl}\text { NAL PROPOSAL NO. } 12 \\ \text { Correspondent } \\ \begin{array}{l}\text { N. W. Reay } \\ \text { Physics Department } \\ \text { Ohio State University } \\ \text { Columbus, Ohio 43210 }\end{array} \\ \text { FTS/Off-net } & \begin{array}{l}614-469-6600 \\ 293-7331\end{array}\end{array}$

A STUDY OF NEUTRON-PROTON CHARGE-EXCHANGE

SCATTERING IN THE MOMENTUM RANGE $50-200 \mathrm{GeV} / \mathrm{C}$

N. W. Reay, K. Reibel, T. A. Romanowski, N. R. Stanton The Ohio State University

M. A. Abolins, M. T. Lin, G. A. Smith

Michigan State University

K. W. Edwards

Carleton University

Engineers - J. S. Fitch, C. J. Rush

The Ohio State University

June 12,1970 
NAL EXPERIMENTAL PROPOSAL

June 12, 1970

\begin{abstract}
A STUDY OF NEUTRON-PROTON CHARGEEXCHANGE SCATTERING IN THE MOMENTUM

RANGE $50-200 \mathrm{GeV} / \mathrm{C}$
\end{abstract}

\title{
ABSTRACT
}

It is proposed to measure the neutron-proton chargeexchange cross-section in an on- line counter experiment for four-momentum transfers between .002 and $1.0(\mathrm{GeV} / \mathrm{c})^{2}$. The experiment will be performed in an intense neutron beam with a continuous momentum spectrum; data will be taken simultaneously for all momenta between 50 and $200 \mathrm{GeV} / \mathrm{c}$. At a beam intensity of 107 neutrons/pulse, the true data-collection rate will be about .5 per pulse. Approximately 90,000 events will be obtained. The vector momenta of both outgoing particles are measured, resulting in a 3 -constraint fit. The two-body topology allows a large rejection of background by the counter $\operatorname{logic}$.

\section{SUBMITTED BY}

N.W. Reay*

K. Reibel

T.A. Romanowski

N.R. Stanton

M.A. Abolins

M.T. Lin

G.A. Smith

K.W. Edwards

Engineers

J.S. Fitch

C.J. Rush
The Ohio State University

Michigan State University

Carleton University

The Ohio State University

* correspondent 


\section{Introduction}

It is proposed to measure the neutron-proton charge-exchange crosssection for incident neutron momenta between 50 and $200 \mathrm{GeV} / \mathrm{c}$, and fourmomentum transfers squared.002 $(\mathrm{GeV} / \mathrm{c})^{2}<|\mathrm{u}|<1.0(\mathrm{GeV} / \mathrm{c})^{2}$.

The np charge-exchange cross-section from .5 to $19 \mathrm{GeV} / \mathrm{c}$ is characterized by a sharp peak at $u=0$, with a width $\delta / \mathrm{u} \mid \cong .02(\mathrm{GeV} / \mathrm{c})^{2}=$ $M_{\pi}{ }^{2}$, rising from a slowly falling background of slope $4-6(\mathrm{GeV} / \mathrm{C})^{-2}$. The behavior is illustrated in Figure 1, which displays preliminary data from a recent experiment (1) performed by members of this group at 2 to $12 \mathrm{GeV} / \mathrm{c}$. Because of its width, the forward peak would seem to relate to pion exchange. However, the amplitude for pure pion exchange is constrained to vanish at $u=0$. Many models, both Regge and non-Regge, have been developed which are based on conspiracy or interference between the pion exchange amplitude and various poles or cuts. These models differ in their predictions about the dependence on incident momentum of the forward peak and the background slope. Naively, effects of pion exchange, falling as the square of the incident momentum, should dominate for small $|u|$; $p$ and $A_{2}$ exchange, falling only as $(1 / P)$, should dominate the crosssection for large $|u|$. As the momentum is increased $\frac{d \sigma}{d u}$ might undergo qualitative changes. The predictions of Williamson(2) (based on a Regge cut mode ${ }^{(3)}$ ) are also shown in Figure 1 . The forward peak which was relatively unchanged between .5 and $19 \mathrm{GeV} / \mathrm{C}$ is predicted to nearly disappear at $200 \mathrm{GeV} / \mathrm{c}$. A wider peak with slope $\sim 17(\mathrm{GeV} / \mathrm{C})^{-2}$ is predicted.

Heuristic arguments and extrapolations based on low energy data must be viewed with a great deal of skepticism. Nevertheless, they emphasize the importance of measuring np charge exchange over as wide a range of 
incident momenta as possible. This, of course, is true for any chargeexchange reaction. However, np charge-exchange is the one most readily extended to high energies - - both because of the extremely simple event topology and because of the possibility of intense high energy neutron beams. This statement is underlined by the fact that it is the only charge-exchange reaction measured with reasonable statistics to the full energy of all existing machines (Serpukhov excluded). Finally, it is one of the very few reactions for which measurements can be made for momentum transfers as small as $|\mathrm{u}|=.002(\mathrm{GeV} / \mathrm{C})^{2}$ for any incident momentum.

Historically, np charge-exchange has proven to be one of the more fundamental and fruitful of all two-body reactions. There is every expectation this would still be true at $200 \mathrm{GeV} / \mathrm{c}$.

11. Experimental Method

A. Plan of Attack. The experiment is performed in an intense beam $\left(\sim 10^{7} /\right.$ pulse) of neutrons having a continuous energy spectrum which peaks near the end point of $200 \mathrm{GeV}$. Data are taken simultaneously over a wide band $(50-200 \mathrm{GeV})$ of the spectrum; this helps to minimize systematic errors in the energy dependence of the cross-section and to maximize the useful beam flux. Charge-exchange events are accumulated at the rate of approximately 0.5 per pulse. The direction and momentum of the fast forward outgoing proton are measured by a magnetic spectrometer, and the velocity and direction of the recoil neutron are measured by a bank of neutron scintillators. Events are processed by an on- 1 ine computer.

There are three kinematic constraints on each event: transverse 
momentum balance, coplanarity, and opening angle. The small transverse components of the proton momentum must be measured very well, especially at small momentum transfer; their accuracy is limited by the uncertainty in the proton direction. There is no constraint on the large longitudinal momentum component of the outgoing proton, which is used to determine the unknown momentum of the incident neutron and need not be measured with extreme accuracy. At high momentum transfer all equations of constraint are limited by the uncertainty in the velocity and direction of the out-going neutron. This can be seen from Figure 4, which displays measurement errors as a function of momentum transfer.

It should be noted that the topology

(incident neutral) $\rightarrow$ (forward charged) + (slow sideward neutral) + (nothing else)

uniquely defines the np charge-exchange reaction, provided that (a) all outgoing particles are detected; (b) both outgoing particles come from the same event (rather than from an accidental coincidence); (c) the incident particle is a neutron; (d) the target particle is a proton. The more securely these topological constraints can be enforced by tight counter logic; the smaller will be the background which the kinematic constraints are asked to reject. It is therefore very important to design an anti-coincidence system with a high efficiency for rejecting extra charged particles and $y$-rays to depress the dominant backgrounds of events which fail to satisfy conditions (a) and (b).

B. Anticipated Difficulties. We believe that the major difficulties to be encountered during the experiment are contained in the following list. Some of the problems have been met and solved in our previous 
charge-exchange work at $12 \mathrm{GeV} / \mathrm{c}$ but are included here for completeness.

1) The detection of efficiency for the slow scattered neutron depends quite strongly on the neutron kinetic energy $T_{n}$, and thus directly on $|u|=2 M_{n} T_{n} ;$ it must be known if systematic errors in the angular distribution are to be avoided. For our previous experiment the counter efficiency was calibrated as a function of energy and bias at a cyclotron, and a Monte Carlo program was written which gives satisfactory agreement with our efficiency measurements and those of other groups for a variety of scintillator geometries.

2) In order to reach small values of $|u|$, outgoing neutrons of very low energy must be detected. The low discriminator bias required invites a large counting rate (and hence accidentals) from room background. By careful shielding and by minimizing the volume of scintillator to be run at low bias we have been able to obtain cross-sections down to $|u|=.002$ ( $1 \mathrm{MeV}$ neutrons). The ultimate limit was set by the large scattering cross-section for low energy neutrons escaping from the hydrogen target; the target for NAL will be half as thick.

3) Both the absolute flux and the spectral shape of the neutron beam must be measured in order to obtain the cross-section as a function of energy. This was done in our previous experiment by measuring the yield of forward elastic scattering in the same beam and using the optical theorem and the known total cross-sections to extract the flux. Such an approach is unnecessary at NAL, since the energy resolution of a total absorption detector (calorimeter) of alternated layers of iron and scintillator is expected ${ }^{(4)}$ to improve with energy, from $25 \% \mathrm{FWHM}$ at $19 \mathrm{GeV} / \mathrm{C}$ 
to better than $10 \%$ FWHM at $200 \mathrm{GeV} / \mathrm{c}$. Such a detector could not operate at the high intensity $\left(10^{7} /\right.$ pulse) necessary for the charge-exchange experiment, so that one or more brief periods of low intensity beam $\left(10^{4}\right.$ to 105 /pulse) will be necessary for studies of spectral shape and calibration of a secondary monitor which can operate at high rates.

(4) The np charge-exchange signal may be as small as $l_{\mu} b$ at 200 $\mathrm{GeV} / \mathrm{C}$; it must be clearly separated from the background by the counter $\operatorname{logic}$ and the kinematic constraints. This problem is discussed in detail in Section 111 .

C. Design of the Experiment. A plan view of the experiment is shown in Figure 2. A beam of $\sim 10^{7}$ neutrons/pulse is produced at an angle of $0-3$ mrad from a point source $\left(<0.2^{\prime \prime}\right.$ diameter) located about 1500 feet upstream. After passing through a lead $\mathrm{Yray}$ filter and a sweeping magnet it is collimated to a divergence of $\pm 2.8 \times 10^{-5}$ radians, filling a $1^{\prime \prime}$ diameter spot as it impinges on the $4^{11}$ long liquid hydrogen target.

The direction of the outgoing forward proton is measured to $\pm 2 \times 10^{-5}$ radians over a lever arm of 100 feet by a system of wire proportional chambers. Because of the high flux $\left(\sim 10^{5}-10^{6} /\right.$ pulse $)$ of charged particles from the target and counters in the beam, ordinary spark chambers with time resolution $\sim 10^{-6} \mathrm{sec}$ would be subject to a large contamination of accidental tracks; the superior time resolution $\left(\sim 6 \times 10^{-8}\right.$ sec $)$ of proportional chambers should eliminate this problem. Two conventional doorframe magnets, each $72^{\prime \prime}$ long with an aperture $24^{\prime \prime} \times 10^{\prime \prime}$ and having a total $\int \mathrm{Bdl}$ of $2600 \mathrm{Kg}$-inches provide a bend of $10^{-2}$ radian at $200 \mathrm{GeV} / \mathrm{c}$. A 20-foot lever arm between the downstream pair of proportional chambers suffices to determine the momentum to $\pm 2 \%$. The transverse momentum is 
then known to $\pm 4 \mathrm{MeV} / \mathrm{C}$ at small $|\mathrm{u}|$ (Figure 4 ).

The direction of the incident neutron must be known to an accuracy comparable to that of the outgoing proton, $\sim 2 \times 10^{-5}$ radians. Since the incident neutron originates from a point source at a known distance its direction may be inferred from the radial position of the interaction in the target, found from extrapolating the proton track.

The slow outgoing neutron is detected in one of 34 neutron scintillators (shown in Figure 3), which are arranged in three banks subtending $10 \%$ of the total azimuth and have an energy-dependent efficiency of $30-70 \%$. The velocity of the neutron is measured by time-of-flight, the azimuthal angle by noting which counter fired, and the production angle by measuring the relative time difference between the light pulses arriving at the photomultipliers at each end of the counter. The size, shape, and distance from the target of the counters in each bank are determined by the energy of the neutrons to be detected: counters for higher energy neutrons require a longer flight path for velocity resolution and a greater thickness for efficient detection; those for low energy neutrons must have a minimum volume and a short time gate to reduce accidentals. The time-of-flight resolution is nearly constant at $\pm 4 \%$. The angle resolution depends on the counter bank (and hence on $|u|$ ) and is shown in Figure 4 ; it is typically $\pm 1^{\circ}$ to $\pm 3^{\circ}$.

It is very important for background rejection to eliminate with the counter logic most of the events having $\gamma$-rays or more than one charged particle. The hydrogen target is therefore surrounded in all directions except those occupled by the outgoing protons by a sleeve and baffles 
("hole counters") of scintillator, and in all directions except those of the outgoing protons and neutrons by lead-scintillator sandwich counters which are $\sim 95 \%$ efficient for $\gamma$-rays. Extra charged particles in the path of the protons will be detected by sampling the ionization in four thin scintillators spaced at intervals between the target and the magnet. The forward cone for $\gamma$-rays will be closed by placing an additional rray veto counter downstream of the magnet and covering its aperture; protons are swept away by the magnet, and a small hole allows the beam to pass through. If necessary, additional sandwich counters may also be placed behind the neutron counters and timed to reject prompt neutrals.

All position information from the proportional chambers, neutron time-of-flight, pulse height, and counter tags will be interfaced to a computer supplied by the user, written on magnetic tape and analyzed between beam pulses. A considerable amount of software already exists as a by-product, of our previous on-line charge-exchange experiment.

\section{II. Background Estimate}

The backgrounds rejected by the counter logic and the kinematic constraints fall into three classes: A) neutron-initiated events which simulate two-body topology; B) random coincidences between the neutron and proton arms; C) two-body events initiated by beam contaminants. Our estimate of the first two classes will draw heavily on our experience at $12 \mathrm{GeV} / \mathrm{c}$.

\section{A. Simulated Two-Body Events. It is difficult to estimate directly} the number of neutron-induced events simulating the topology of np charge- 
exchange, since such evenis invoive a failure of the anticoincidence system. It is easier -- and we feel more believable --- to extrapolate from our experience at $12 \mathrm{GeV} / \mathrm{c}$ than to construct a large and artificial Monte Carlo program. We make the following assumption: The anticoincidence system and the proportional chambers are able to reject events with multiple charged prongs down to a level small compared with events having only one charged prong which proceeds forward with a momentum greater than $30 \mathrm{GeV} / \mathrm{c}$. We then assert that these high-momentum singleprong events must have come from a process in which charge was exchanged.

Consider a model in which projectile and target nucleons pass one another, either or both becoming "excited" and subsequently decaying into a nucleon plus pions. Unless charge is exchanged, decays coming from the excited "beam-1ike" particle will be peaked forward and contain an even number of charged particles. Evidence that this assertion is valid comes from the fact that at $12 \mathrm{GeV} / \mathrm{c}$, the rate of events in the proton arm which survived the veto by the (crude) anticoincidence system was only a factor of four higher than the chärged flux expected from np chargeexchange alone.

If the ma in contributors to the singly-charged forward flux are (inelastic) charge-exchange reactions, such processes ought to decrease with incident momentum at least as fast as $1 / P$, while np charge-exchange appears at most to fall off as $(1 / P)^{1.5}$. (We neglect high-mass inelastic charge-exchange channels opening at higher energies. They will be eliminated by their high multiplicity and/or large transverse momentum.) We therefore predict that the ratio of inelastic background to chargeexchange signal should be increased by $(200 / 12)^{1.5-1.0}=4.1$ over its 
value at $12 \mathrm{GeV} / \mathrm{c}$, which was $2-5 \%$. We have sharpened the resolution of our kinematic constraints by an overall factor of four from that experiment; the improved anticoincidence system should provide an additional large rejection factor.

B. Random Coincidences. The high intensity of the neutron beam will cause random coincidences between the neutron and proton arms, increasing the trigger rate. Moreover, those randoms which pass the kinematic constraints can give rise to a false sharp forward peak: Since $|u| \propto$ (neutron kinetic energy) $\propto(t i m e-o f-f l i g h t)^{-2}$, a flat time distribution will give $\frac{d \sigma}{d u} \propto|u|^{-3}$. The random rate is proportional to the length of the time gate $(\sim 100 \mathrm{nsec})$, to the neutron counter singles rate, and to the rate of proton arm events surviving veto by the anticoincidence system. By the arguments of the preceding section, the latter rate may be estimated by scaling our observed rate at $12 \mathrm{GeV} / \mathrm{c}$ by the ratio of beam intensi.ties, solid angles, and by the inverse of the momentum. We obtain $250 /$ pulse.

The singles rates in the neutron counters arise primarily from beam-associated reactions occuring in the liquid hydrogen target or nearby surrounding material. General "room background" may be el iminated by suitable shielding. The main source of singles background in the $12 \mathrm{GeV} / \mathrm{c}$ experiment was interactions of the neutron beam halo in the dense materials near the liquid hydrogen flask. A new beam collimating system, coupled with a less massive target container and the proposed tighter anti-counter system, should drastically reduce both halo and the probability for halo-induced events to go undetected. 
About $10 \%$ of the random singles came from interactions in liquid hydrogen. These cannot be eliminated, and must be scoled with beam intensity $(\times 20)$ and scintillator area $(\times 2)$, yielding $6.4 \times 10^{4}$ counts/ pulse in the neutron counters.

Assuming a duty factor of 0.5 , the derived random rate is $3.2 / \mathrm{pulse}$ or about 6 times the true event rate. Random rates 60 times the true rate will give a "post-analysis" background signal equal to the true signal in the lowest momentum transfer bin.

C. Beam Contamination. Passing the beam through a lead filter and a sweeping magnet prior to collimation will reduce the total flux of $\psi$-rays and charged particles to a negligible level compared to the neutron flux, and a thin anticoincidence counter in the beam upstream of the hydrogen target will reject events initiated by the surviving charged particles. Above $50 \mathrm{GeV} / \mathrm{c}$ the anti-neutron flux should be vanishingly small, and the residual $\mathrm{y}$-ray flux is harmless. However, the flux of $\mathrm{K}^{\circ} \mathrm{L}$ predicted by Hagedorn and Ranft (5) at $50 \mathrm{GeV} / \mathrm{C}$ is $17 \%$ of the neutron flux at that momentum. At lower momenta the ratio of $K^{\circ}{ }_{L}$ to neutron flux is even higher. The kinematic constraints are not able to separate $K^{\circ} L^{P}$ $\rightarrow \mathrm{K}^{+} \mathrm{N}$ from $\mathrm{np}$ charge-exchange. For this reason, provision will be made to include an 8-meter gas threshhold Cerenkov counter in the proton arm to study this potential source of background (see the Appendix).

IV. Rates

The rate for $n p$ charge-exchange is estimated for both 0 mrad and 3 mrad production angle neutron beams, using the formula

$\frac{\text { events }}{\text { pulse }}(p)=I(p) N \sigma_{n p}(p) \frac{\delta \Phi}{2 \pi} \overline{\overline{E f f}}_{N}$ 
where $I(P)$ is the neutron beam as a function of momentum, $N$ the number of protons per $\mathrm{cm}^{2}$ in the target, $\sigma_{n p}(P)$ the total np charge-exchange cross-section, $\frac{\delta \varnothing}{2 \pi}$ the fractional azimuthal acceptance, and $\overline{E f f}_{N}$ the mean neutron counter efficiency.

The beam intensity may be derived from the formula of Hagedorn and Ranft ${ }^{(5)}$, using $3 \times 10^{12}$ protons interacting per pulse, projecting neutrons onto a 1 inch diameter spot 1500 feet downstream of the production target. An attenuation factor of .5 is assumed due to lead in the bean. The calculated integral flux is $7.2 \times 10^{7}$ neutrons/pulse at $0 \mathrm{mrad}$, $1.9 \times 10^{7}$ neutrons/pulse at $3 \mathrm{mrad}$. In order to be conservative in estimating intensities which can be achieved and tolerated, the beams shown in Figure 5 and used in the calculations are normalized to $1 \times 10^{7}$ neutrons per pulse.

$$
\begin{aligned}
N= & 4.2 \times 10^{23} \text { protons } / \mathrm{cm}^{2} \\
\sigma_{n p}(P)= & \left(1.4 \times 10^{-28} \mathrm{~cm}^{2}\right)(10 / P)^{1.5}, \text { derived from the } 12 \mathrm{GeV} / \mathrm{c} \\
& n p \text { charge-exchange experiment. } \\
\delta \varnothing / 2 \pi= & 0.1 \\
\overline{\mathrm{Eff}}_{\mathrm{N}}= & 0.5
\end{aligned}
$$

Using the above, we calculate the rates shown in Figure 5 . The integral event rate in the momentum range 50 to $200 \mathrm{GeV} / \mathrm{c}$ is 0.5 events/ pulse for both 0 and 3 mrad beams.

If the data are binned in $10 \mathrm{GeV} / \mathrm{c}$ bins, it would take 90,000 events to gather statistics per bin equivalent to each of the data curves shown in Figure 1. At 10,000 pulses/day, this would require 18 days of data taking. Tuning will require an additional 20 days, most of which could be spent under low or intermittent beam conditions. 
These time estimates are meant to be conservative, and are based in part on actual tuning and running time for the $12 \mathrm{GeV} / \mathrm{c}$ experiment. V. Special Equipment and Services

A. Furnished by the User:

1) All scintillation counters (including neutron counters) and proportional chambers.

2) An on-line computer system complete with interfacing and software.

3) The neutron calorimeter for monitoring the beam flux and spectrum shape.

B. Furnished by NAL:

1) Construction of the collimator and channel for a high intensity neutral beam. The collaboration making this proposal would be willing to devote one physicist full time to aid in the design and construction of such a beam. It would desire also to be a part of any effort involved in extraction of the neutrons and measurement of the momentum spectrum.

2) A magnet of at least $1300 \mathrm{Kg}$-inches is needed for sweeping charged particles from the beam. The magnet could have a vertical and horizontal gap as small as $2^{\prime \prime} \times 2^{\prime \prime}$.

3) A 4" long $\times 2^{\prime \prime}$ diameter liquid hydrogen target. The vacuum jacket must be designed to leave the minimum amount of material in the region of the beam halo ( $\sim 6^{\prime \prime}$ radius).

4) Magnets totalling $2600 \mathrm{Kg}$-inches of bending for momentum analys is. of the outgoing proton. The experiment is designed around two 72 " doorframe magnets with a $10^{\prime \prime} \times 24^{\prime \prime}$ aperture. The requirements on field uniformity are not very severe; it should be flat to better than $15 \%$ over the useful aperture.

5) Construction of the bunker for shielding the neutron counters. 


\section{BIBLIOGRAPHY}

1) E.L. Miller et al, Bull. Am. Phys. Soc. 15, 660(1970)

2) G.T. Williamson, private communication

3) See e.g. F. Henyey et al, Phys, Rev. 182, 1579(1969)

4) L.W. Jones, Neutral hadron Detectors for NAL, National Accelerator Laboratory 1969 Summer Study Report SS-88, Vol.111

5) R. Hagedorn and J. Ranft, Suplemento Al Nuovo Cimento, Ser. I, 6, 169(1968); M. Awschalom and T. White, Secondary Particle Production at $200 \mathrm{GeV}$., National Accelerator Laboratory FN-191 (1969)

6) P. Astbury et al, Phys. Letters 16, 328(1965); 23, 396(1966); also results of $D$. Cline et al reproduced in $B . J$. Hartley et al, Phys. Rev. 1D. 954(1970). 


\section{Appendix: $K^{\circ} \mathrm{P}$ Charge Exchange}

Background rates for the reaction $K^{\circ} L^{P} \rightarrow K^{+} N$ may be estimated under the assumption $\sigma_{K^{\circ} P} \rightarrow K^{+} N=0.5 \sigma_{K^{-} P} \rightarrow \bar{K}^{\circ} N=\left(3.3 \times 10^{-29} \mathrm{~cm}^{2}\right)(10 / P)$. The assumption checks within $30 \%$ at $3-5 \mathrm{GeV} / \mathrm{c}$, the highest momenta for which $K^{+} N \rightarrow K^{\circ} P$ data exists. (6) " $K^{-} P$ charge-exchange data exists to $12 \mathrm{GeV} / \mathrm{c}$. (6) Background from the $K^{\circ}{ }_{L} P$ reaction is estimated to be as high as $10-20 \%$ in the 40 to $60 \mathrm{GeV} / \mathrm{c}$ range, and so will have to be investigated. If in studying this background reaction the $\mathrm{K}^{+}$signal proves to be clean, it would be possible to measure $K^{\circ}{ }_{L} P$ charge-exchange using the same equipment in a different geometry. The integral event rate predicted for a $3 \mathrm{mrad}$ beam is $0.1 / \mathrm{pulse}$ in the 10 to $60 \mathrm{GeV} / \mathrm{c}$ range, normalized to $10^{7}$ neutrons/pulse. 


\section{LIST OF FIGURES}

Figure 1. Differential Cross-Sections for Neutron-proton Charge-exchange.

Figure 2. Plan View of the Experiment

Figure 3. Neutron Counter Arrangement

Figure 4. Experimental resolution and Kinematic Constraints

Figure 5 Neutron Fluxes and Expected Charge-exchange Yields for 0 and $3 \mathrm{mr}$ Beams 


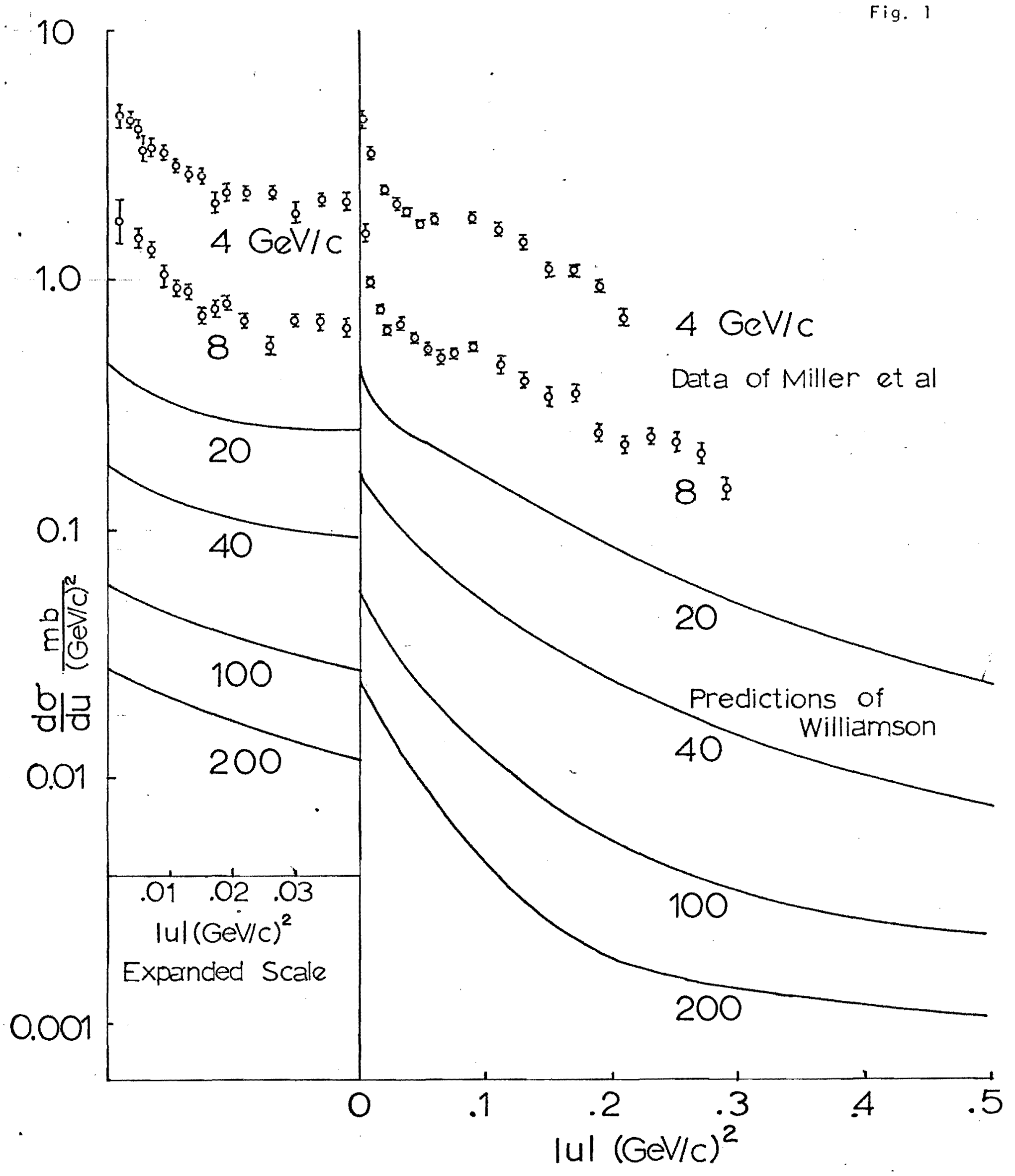


MWPC $_{i}$ - MULTIWIRE PROPORTIONAL CHAMBER MODULES

$A_{i}$ - "HOLE COUNTERS"

$\mathrm{H}_{\mathbf{i}}$ - SCINTILLATOR HODOSCOPES

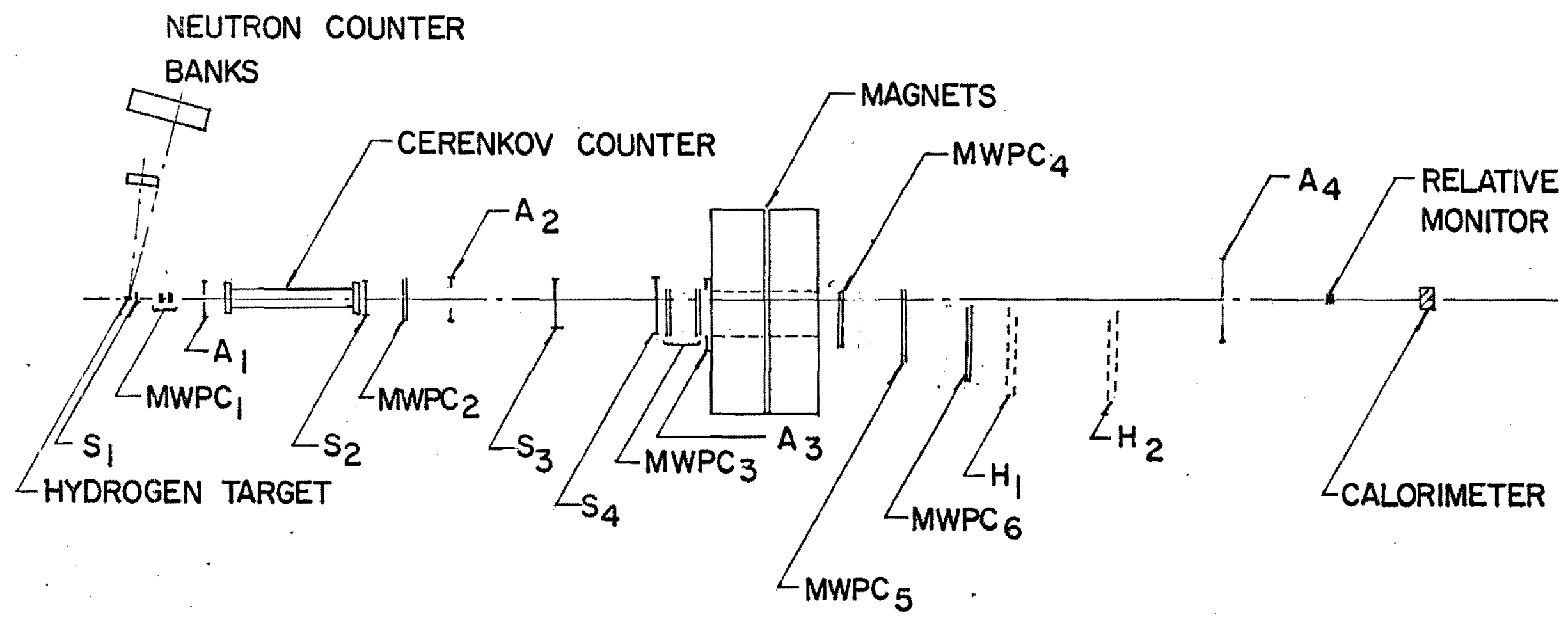

HORIZONTAL' SCALE: $\stackrel{0}{0} 10 \mathrm{ft}$.

VERTICAL SCALE: $10 \mathrm{ft}$. 

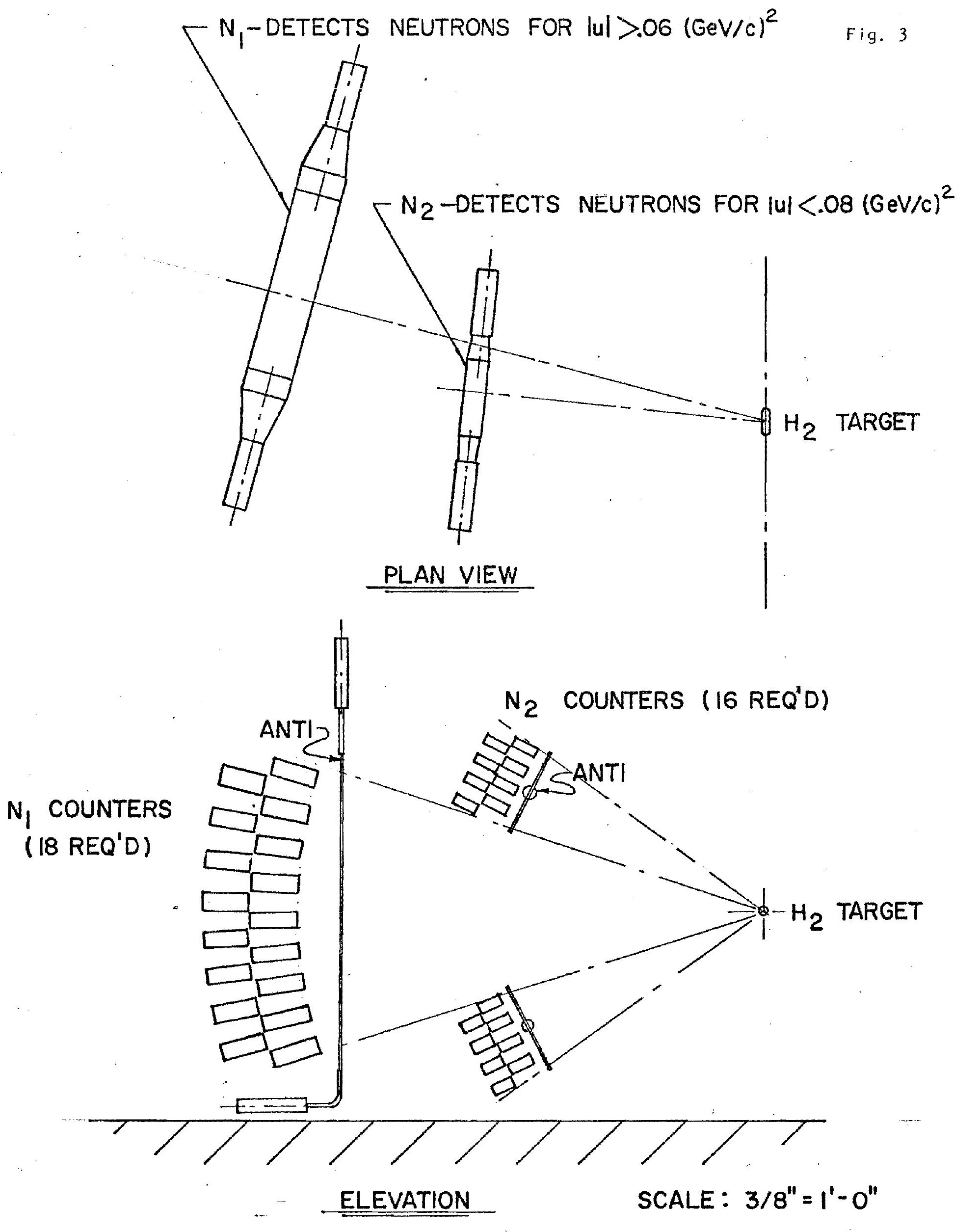

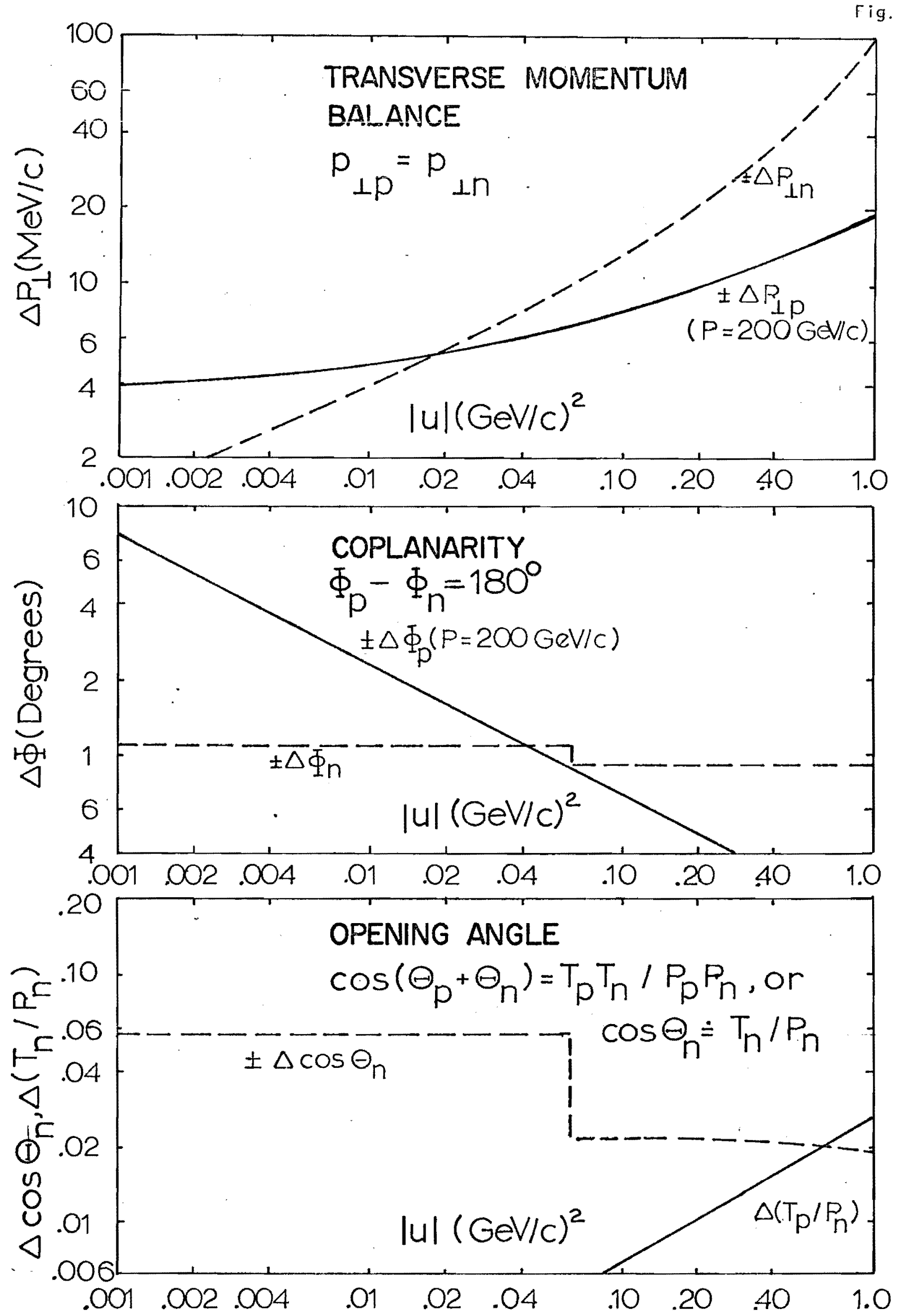


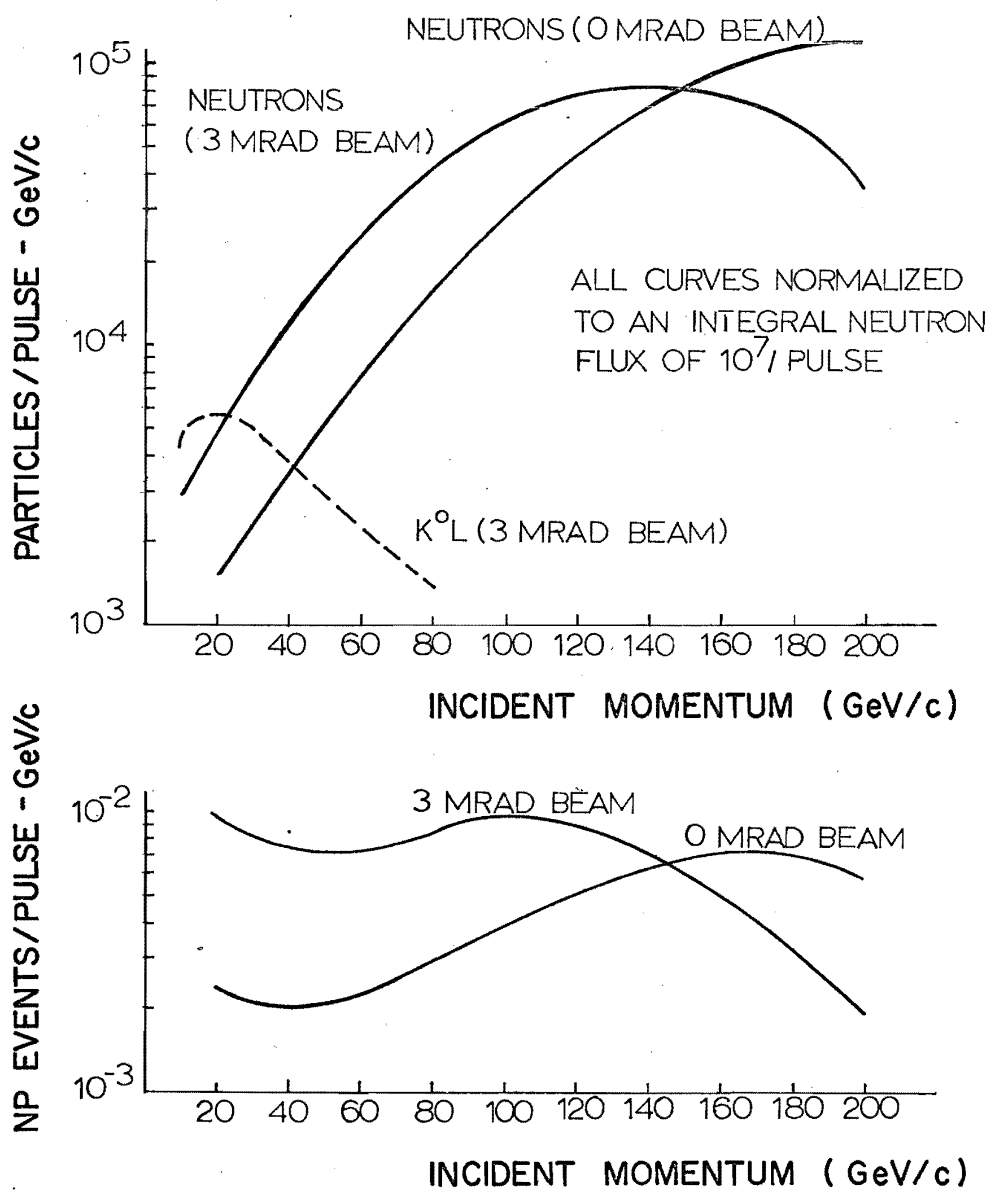

